\title{
Estrategias para la transformación de la gestión educativa tomando en cuenta la recuperación del sujeto
}

\section{Strategies for the transformation of educational management taking into account the recovery of the subject}

\author{
Cinthia Gimena Vargas Cayetano \\ cindiabril1234@gmail.com \\ Código ORCID: 0000-0002-1986-527X -
}

\author{
Orlando Torrico Rocha \\ cindiabril1234@gmail.com \\ Código ORCID: 0000-0003-1611-4056
}

Universidad Pedagógica ESFM “Simón Rodríguez”, Cochabamba, Bolivia

Recibido octubre 2020 / Arbitrado noviembre 2020 / Aceptado diciembre 2020 / Publicado enero 2021

\section{Resumen}

En el presente trabajo de sistematización se presentan las experiencias vividas de un equipo de docentes investigadores en el marco del Modelo Educativo Sociocomunitario Productivo MESCP con la intención de mejorar el ambiente laboral de las instituciones educativas Bolivia Japón y Alto San Pablo en el distrito de Puerto Villarroel y Cochabamba 1 y 2. Es una investigación cualitativa y de campo porque los docentes fueron partícipes directos en el hecho de estudio y basándose en sus experiencias, realizaron los diálogos pedagógicos en los que se explican los problemas de convivencia de ambas instituciones y las soluciones propuestas para mejorarla. Además, se reflexiona sobre la práctica que desempeña el gestor en una institución educativa. Se concluye que el gestor educativo junto con el docente, son los responsables principales en la comunicación asertiva de la institución y la convivencia educativa.

\author{
Palabras clave: \\ convivencia \\ educativa; gestión \\ educativa; \\ relaciones \\ humanas; diálogo \\ pedagógico; \\ educación \\ participativa
}

\begin{abstract}
In this systematization work, the lived experiences of a team of research teachers are presented within the framework of the MESCP with the intention of improving the work environment of the educational institutions Bolivia, Japan and Alto San Pablo in the district of Puerto Villarroel and Cochabamba 1 and 2. It is a qualitative and field research because the teachers were direct participants in the study and based on their experiences, carried out the pedagogical dialogues in which the problems of coexistence of both institutions and the solutions proposed to improve it are explained. In addition, it reflects on the practice carried out by the manager in an educational institution. It is concluded that the educational manager, together with the teacher, are the main responsible for the assertive communication of the institution and the educational coexistence.
\end{abstract}

\author{
Keywords: \\ Educational \\ coexistence; \\ educational \\ management; \\ human relations; \\ pedagogical \\ dialogue; \\ participatory \\ education
}




\section{INTRODUCCIÓN}

L os procesos de transformación educativa deben enfocarse a dar respuestas a los problemas y necesidades que atañen a la población estudiantil y la comunidad. Anteriormente la educación era verticalista, autoritaria, cuantificadora; el estudiante tenía que aprender y el profesor enseñar, mientras que el director debía solo mandar y ordenar fríamente. Sin embargo, hoy el Modelo Educativo Sociocomunitario Productivo (MESCP), plantea otra forma de hacer gestión y una nueva manera de educar partiendo de la vida, en la vida y para la vida, buscando que los mismos sujetos sean quienes, en un trabajo en conjunto puedan transformar las diferentes realidades del contexto que les rodea.

Partiendo de esta idea, la comunidad pedagógica de transformación para la gestión educativa, que está conformada por maestras y maestros con diferentes realidades, donde se presentan el distrito de Puerto Villarroel y el distrito de Cochabamba 1 y 2 estuvo orientada en esta labor al estudio de las problemáticas y la realidad del contexto en el que se desenvuelve la comunidad, lo que ha permitido observar dificultades y potencialidades que se presentan en torno al logro de la transformación educativa.

Por consiguiente, en el presente trabajo de sistematización se describen las experiencias vividas de un equipo de maestros y maestras, en el marco del MESCP, que permitirán buscar la transformación, o en su caso, el cambio de la realidad, pero sobre todo la reflexión orientada a la práctica adecuada que desempeña un gestor en la institución educativa.

Proporcionalmente se presenta el análisis de la implementación de distintas actividades, dinámicas y estrategias planteadas con la visión de fortalecer las relaciones institucionales de un determinado contexto educativo con el fin de optimizar las relaciones entre administrativos, docentes y directivos. Esto con la intención de influir positivamente la práctica pedagógica.

En el caso de La UE “Bolivia Japón” visitada por la investigadora Cinthia Gimena Vargas Cayetano, se observó una problemática en el ambiente educativo de la institución en torno al relacionamiento entre todos los participantes de proceso educativo. En el plantel se evidencia una falta de socialización y apoyo mutuo entre el personal 
docente que no puede ser tratado adecuadamente según el reglamento interno.

Cabe acotar que el divisionismo entre maestros en la comunidad educativa perjudica las buenas relaciones interpersonales entre colegas y administrativos, por ende, repercute en los padres de familia perjudicando en gran medida la efectividad en el cumplimiento de las actividades programadas. Esto se debe a que algunos maestros son indiferentes, lo cual se nota en su actitud durante las actividades que se realizan en la institución como horas culturales, actos cívicos y en los trabajos de las comisiones. Por esta razón no se efectiviza lo planificado. Esta situación preocupa al gestor porque, para que una institución alcance sus objetivos, es importante la unidad de los maestros, el trabajo en equipo, la coordinación del director con los docentes, los padres de familia y autoridades.

Desde la perspectiva de la transformación educativa, el gestor de la institución debe crear un clima de criterio y madurez para resolver en conjunto y coordinar los desafíos y problemas que se presentan a lo largo de la gestión, logrando un compromiso activo y constructivo de manera que la institución educativa tenga la fuerza que le ayude a mejorar y conseguir la solución del problema que acontece. Como hace referencia el Modelo Educativo Sociocomunitario en el Estado Plurinacional, el gestor debería ser una persona conocedora en todo lo que compete en la educación, llevar su gestión con capacidad de conocimiento y creatividad porque tiene la obligación de orientar a los maestros y padres de familia sobre la función que tiene el proceso educativo brindando espacios de participación en actividades, colaboración, convivencias y debe estar abierto al diálogo y a la escucha para involucrar a todos los actores de la comunidad.

En este orden de ideas, la educación se construye con la participación de los actores de la unidad educativa, y a su vez la educación contribuye a la conciliación del modelo 070 a través de la formación integral y holística de estudiantes con pensamiento crítico y acción transformadora, propositiva, con la práctica de valores sociocomunitarios y estableciendo un diálogo intercultural e interreligioso entre los maestros.

Por otro lado, en el caso de la segunda institución que se estudió en esta experiencia, la Unidad Educativa "Alto San Pablo" visitada por los investigadores Orlando Torrico Rocha y Cesar 
Vargas Cayetano, se evidencia la misma problemática, es decir, un ambiente de trabajo un tanto complicado, ya que el gestor es autoritario en algunas ocasiones, el equipo está dividido por grupos de maestros apegados al gestor, algunos grupos de profesores que manifiestan empatía y otros que no, lo cual se observa más en los momentos del recreo, acontecimientos cívicos y actividades extracurriculares.

De este modo, desenvolverse en medio de una realidad divisionista y con la prima del sentido individualista no es favorable para trabajar comunitariamente. Sin una buena relación es complicado lograr objetivos con un horizonte común. Sin embargo, cada uno está poniendo de su parte intentando que todo funcione correctamente. Además, la colaboración entendida como esa acción de ayuda recíproca desinteresada, que vela por el bienestar común de dicha comunidad es escasa. En lugar de confianza, hay recelo e individualismo, sectarismo, el grupo es reacio e indiferente a las realidades.

Es por tanto que se puede decir que falta desarrollar en esta institución las buenas relaciones para encontrar el sentido a la práctica educativa y su orientación comunitaria, es decir que cada sujeto desde su particularidad colabore en los procesos de transformación pedagógica.

En concordancia con la ley 070 Avelino Siñani y Elizardo Pérez (2010), el rol del gestor como la cabeza de las instituciones, puede motivar y direccionar ampliamente, seguido de los maestros/as convencidos de su rol, comprometidos en la transformación de la comunidad. En esta ley se recalca que el gestor debe tener una capacidad amplia de relacionamiento, de visión, organización, de trabajo en conjunto, compromiso, convencimiento, estar en posición de una autoridad pedagógica, estar inmerso de manera cercana a los maestros, y comunidad entera, logrando el sentido y la importancia de tener un horizonte común. Pero este rol no se evidencia en la institución.

Otro aspecto no favorable en la situación es la falta de diálogo y de compromiso con sentido de su gestión por parte del gestor de la institución ya que no respeta las decisiones acordadas en consenso, impone sus ideas y mantiene una amenaza constante mediante sanciones a quien no esté de acuerdo con dichas ideas. En consecuencia, se devalúa la calidad de las relaciones sociales 
en el plantel y se desvirtúan los procesos de educación que se establecen en el MESCP.

Cabe destacar que en dicho modelo (MESCP) se amplía el campo de estudio laboral y personal tanto para los maestros, estudiantes, padres de familia y la comunidad en general, ya que en este nuevo paradigma educativo todos los conocimientos que se desarrollan están más enfocados en la recuperación de conocimientos, mitos, religiones y tradiciones culturales del país y no como se hacía en años anteriores con las leyes de educación que eran copiadas de otros países, porque las autoridades que regían el gobierno en años anteriores solo apreciaban lo extranjero y buscaban la conversión a los modelos de vida importados causando que en todo el país, las personas fueran perdiendo su identidad. En cambio, en este nuevo modelo se da un mejor enfoque y visión a toda la comunidad educativa en la formación integral de los estudiantes.

Dadas las circunstancias antes descritas, la presente investigación se propuso objetivos como: Mejorar el clima de convivencia institucional mediante actividades de calle y salidas de campo, se llamó al diálogo pedagógico entre docentes y gestores, se propuso desarrollar y fortalecer cualidades como la autoestima y realizar talleres y charlas de relaciones humanas que incentiven a la unión y actividades que inciten a la sociabilización entre el personal.

En este sentido, una de instituciones que participó en esta experiencia fue la Unidad Educativa "Bolivia Japón" que fue visitada por la investigadora Cinthia Vargas Cayetano. La misma se encuentra ubicada en la zona Sur denominada Lacma, específicamente en el barrio "San Juan Bosco" de la Ciudad de Cochabamba, perteneciente al distrito 5 Sub alcaldía Alejo Calatayud y al sistema educativo regular.

Dicha institución atiende el nivel primario, es de fácil acceso ya que hay bastantes líneas de transporte que pasan por la escuela. Asisten estudiantes de diferentes lugares ya que, al ser una escuela pequeña, trabaja con estudiantes que presentan diferentes problemas de conducta en el aprendizaje. La mayoría de los estudiantes son de familias de condición social media, esto ocasiona que el trabajo con todos ellos sea difícil, los padres de familia son tranquilos, pero existe siempre un padre que trata de ocasionar algún daño a la unidad educativa. 
En cuanto a la segunda institución, la Unidad Educativa "Alto San Pablo" visitada por los investigadores Orlando Torrico Rocha y Cesar Vargas Cayetano, se encuentra en la población con el mismo nombre. Cuenta con una infraestructura propia, debidamente equipada con 13 aulas, dos tinglados, raqueta frontón, sala de computación, banda de guerra entre otros. Es dependiente de la Dirección Distrital de Educación Puerto Villarroel, provincia de Carrasco del departamento de Cochabamba con la Unidad Educativa de Padilla como única asociada ya que las otras escuelas tuvieron que cerrarse por falta de estudiantes y alimentación.

Así mismo, la mayor parte de la población está formada por habitantes que migraron de diferentes partes del país, por esta razón los idiomas más hablados son el quechua y el castellano, además de algo de aimara y guaraní. La economía de la población se basa principalmente en el cultivo de la hoja de coca, plantas frutales de la zona, yuca y también las plantas medicinales e industriales, las cuales no son solo para el consumo interno ya que los excedentes se venden a las poblaciones más cercanas. También existen otras actividades económicas, como: la crianza de ganado vacuno, ovino, porcino, avícola y últimamente la piscicultura. En el aspecto religioso, existen católicos que aún mantiene costumbres culturales como la challa a la madre tierra, la k'oa el primer viernes de cada mes, pero la mayoría profesan su fe en Dios perteneciendo a la Iglesia evangélica.

\section{METODOLOGÍA}

L a presente investigación está basada en diálogos pedagógicos

a partir de la experiencia que tuvieron los investigadores durante su participación en las instituciones antes descritas. Sin embargo, se puede decir que esta investigación concuerda con el tipo cualitativo porque no emplea algún método de análisis estadístico ni de variables y es descriptivo e interpretativo porque se enfoca en el relato de situaciones con un fin reflexivo.

También está enmarcada dentro de un modelo de campo porque los investigadores fueron partícipes directos del hecho de estudio y además, recolectaron los datos mediante la observación participante. Finalmente, para el análisis e interpretación de los resultados se emplearon diálogos interpretativos y reflexivos con el equipo de trabajo a modo de retroalimentación, donde todos los 
participantes expresaron sus conclusiones. En palabras de los mismos investigadores se puede apreciar lo siguiente:

Por lo mencionado líneas arriba y con el fin de poder compartir nuestra experiencia en el análisis y reflexión de nuestra realidad, el presente trabajo de sistematización se encuentra estructurado en base a lo siguiente: Las experiencias individuales de la gestión educativa, donde se observará el relato de cada integrante de la CPTGs; luego se referirá a la reflexión comunitaria de los avances, problemas y propuestas para la gestión de las unidades educativas, la cual expresará los avances, problemas, propuestas y una reflexión colectiva de la comunidad de gestión educativa; para terminar de plantearemos conclusiones y propuestas que sirvan para fortalecer la gestión educativa en nuestro departamento.

\section{RESULTADOS}

1 n la Unidad Educativa Bolivia Japón, el director observó la gestión anterior y evidenció que en la institución existen divisiones que están dañando el clima en la escuela, es por eso que en esta nueva gestión escolar se realizó una reunión de profesores donde se determinó que cada comisión trabajaría para tratar de mejorar el clima institucional.

Así pues, la comisión social se organizó dando lectura a su propuesta de trabajo para realizar algunas actividades en el transcurso del año. Del mismo modo la comisión disciplinaria comenzó dando su lectura de su propuesta de trabajo entregando reglamento interno para los estudiantes, maestros y padres de familia. Cada reglamento debía ser leído en las reuniones con los padres de familia y en la reunión de profesores según las actividades por bimestre. Al leerse el trabajo de la comisión disciplinaria, se dieron a conocer las impuntualidades e inasistencias injustificadas de algunos docentes, éstas se cancelarían de forma interna mediante sanciones económicas. También se habló sobre el uso de los uniformes de los lunes y los viernes y todos los participantes estuvieron de acuerdo con lo planteado.

Sin embargo, este acuerdo no se cumplió de manera eficiente. La investigadora pudo observar que algunos profesores se percataban solamente de aquello que les convenía. Los registros 
en los cuadernos de asistencia y otras actas eran constantemente alterados a favor o en contra de unos cuantos. En pocas palabras, no había un sistema honesto e igualitario de control. Eso por mencionar solamente uno de los problemas en el plantel. En el análisis que confiere al campo de la disciplina y la responsabilidad, se pudo observar que los profesores no estaban interesados en seguir las normas acordadas en la reunión.

Otro factor que se pudo observar fue el asunto de la "preferencia" lo cual fue motivo de discordia. El director en una reunión de profesores, informó la molestia que tuvo con algunos de ellos que se atrevieron a decir que él tenía preferencia por determinados profesores. También le reclamaron que "él es bueno con los que lo acompañan en las copas". El director molesto informó en la reunión lo que estaba ocurriendo y que él se sentía muy triste por la forma en que fue tratado. Este problema se originó por la cancelación de las sanciones de retrasos, faltas y uniformes que se habían acordado al principio. En consecuencia, se llegó de nuevo a otro acuerdo en el que la sanción no fuera por descuento sino por memorándum. El director un poco molesto vio de mejor manera la determinación, aunque algunos no se quedaron conformes.

Pero este no fue el único altercado que se evidenció durante el estudio. También se pudo ver que en otros eventos institucionales se producían riñas o falta de apoyo entre los colegas. Por ejemplo, en las actividades de carnaval, actos deportivos y otros eventos culturales. Parecía más bien que "cada uno quisiera dañar al otro"

Como respuesta a esta situación, se trató de unir a todos los colegas con una serie de actividades en la escuela, pero algunos no participaron. La comisión preparó para el día de la amistad una reunión para que los profesores socializaran. Se preparó un plato y la comisión regaló unos recuerdos a todos los docentes. En ese momento se puedo observar que todos estuvieron riendo, pero saliendo del aula todo volvió a ser lo mismo, no hablaban entre ellos, ni se miraban si quiera. La división entre los colegas provocó que el director tuviera que trabajar con dos grupos por separados para evitar discusiones y quejas.

Como continuaban los conflictos que además estaban afectando los eventos institucionales, la comisión social realizó otra actividad para tratar de unir a los colegas y fue una salida de paseo. Todos fueron de viaje a Inca Chaca, y como de costumbre 
cada uno por su lugar. A pesar de las parcelas, todos caminaron, observaron y platicaron al menos por el tiempo que duró la actividad. Luego, el director convocó a una reunión a todos los profesores donde les pidió tratar de mejorar el ambiente institucional y ninguno dijo algo relevante.

Fue así como surgió otra propuesta para mejorar el clima institucional. Esta vez mediante un taller de relaciones humanas. El director estuvo de acuerdo y comenzó a desarrollar el taller y la licenciada convocó a todos a participar en las actividades de convivencia. Como resultado del taller, muchos coincidieron en cambiar y olvidar ese divisionismo existente en la escuela.

Seguidamente la comisión social propuso otra actividad llamada abrazo terapia. Dicha actividad sacó a los participantes de su zona de confort porque, según la investigadora: "era muy difícil acercarse y recibir con un abrazo a los colegas, así que optamos primero con el saludo de mano, aunque no estábamos acostumbrados a esa forma de saludo" Por esa razón, decidieron saludar a los colegas más conocidos para comenzar. Según lo relatado por la investigadora más adelante se puede evidenciar que el contacto físico les resultó complicado de lograr: "nos resultó un poco difícil acercarnos a algunos profesores porque se iban rápido como para hacernos entender que no querían saludar de esa forma, sin embargo, ya están acostumbrándose a esa forma de saludo..."

Más adelante se propuso otra actividad enfocada al compartir mediante alimentos durante el recreo, pero no tuvo tanto éxito. La investigadora relató lo siguiente:

...otra actividad que hicimos fue invitar en los recreos algún producto a todos los profesores ya sea un dulce, un chocolate, un pancito y así poder cambiar la monotonía de entrar y salir de la escuela, esta actividad se cortó porque no todos traían el producto y para que no retrocediera lo dejamos así.

Al finalizar el período de investigación se lograron algunos avances en la convivencia escolar. Sin embargo, el conflicto persiste por lo cual se propone continuar con esta gestión en el plantel. Según lo relatado por la investigadora: 
"Todos los profesores vemos que por los menos ya cambió el ambiente en la escuela, pero todavía hay profesores que tratan de amargar ese ambiente. Y lo que queremos hacer es volver a realizar otro taller de relaciones humanas para que así logremos nuestro objetivo de llevarnos bien todos como una familia en la escuela."

Por otra parte, en la Unidad Educativa "Alto San Pablo" con un problema similar de relacionamiento social, los resultados de la experiencia indicaron que ésta se origina en la carencia de espacios para la convivencia y fraternización en la comunidad educativa, aunado esto al modelo vertical de gerencia tradicionalista y de jerarquías que se sigue aplicando en el plantel. Por esta razón se complican las relaciones sociales entre los participantes del hecho educativo comenzando por las autoridades quienes han incurrido inclusive en el abuso de poderío.

En la búsqueda de solución a esta problemática, se socializó en reuniones con los padres de familia y toda la comunidad educativa de Alto San Pablo y se dieron a conocer las expectativas, deseos y esperanzas, respecto de la educación, la escuela y la formación de los educandos. Se conversó sobre el sentido y la importancia de las líneas estratégicas desarrolladas desde el ministerio de educación, y así poder responder a esas expectativas planteadas donde se priorizó la siguiente línea de acción: Educación participativa. La misma se articula al trabajo curricular inserto en las planificaciones que se presentan mensualmente a través de diferentes contenidos relacionados con el entorno natural y social de las comunidades, de este modo el proceso de enseñanza y aprendizaje se base en una participación activa, democrática y consensuada. Según lo relatado por el investigador Orlando Torrico Rocha, en la situación se pudo observar lo siguiente:

Luego de haber dialogado de manera participativa, propositiva y reflexiva, vemos que en nuestra comunidad de trabajo existen pocos espacios de relacionamiento con los maestros y directores, ya que ciertas autoridades aún se desenvuelven en el marco de una gestión tradicional, donde no se hace énfasis al sujeto, sino que se mide la excelencia enmarcada al cumplimiento de deberes y en algunas ocasiones se manifiesta el abuso de autoridad. La creación de grupos 
desfavorece una relación buena y saludable, ya que en ella se puede incurrir a la separación y creación de rivalidades... un malestar general en la comunidad educativa.

Fue así como el eje priorizado en la educación participativa, se dio en base a los pocos espacios de participación en el proceso de aprendizaje de los educandos y la poca presencia en las actividades extracurriculares del colegio. Muchos padres de familia se no se integran al proceso de educación de sus hijos alegando que no saben asesorarlos porque nunca fueron a una casa de formación y no tienen tiempo. Sin embargo, entendiéndose el sentido y la gran importancia de que ellos son parte de esta idea común en el proceso de seguimiento y apoyo moral de sus hijos/as, se toma las siguientes acciones en el trabajo curricular, sale de ellos el compromiso de dedicarles tiempo a sus hijos/as y preguntarles por ejemplo ¿Cómo te ha ido? ¿Qué aprendiste? ¿Qué tarea tienes? ¿Qué te cuesta más? ¿Qué te gustó más hoy?

A pesar de que los padres de familia se excusan en la idea de que no están capacitados para guiar a sus hijos en el aprendizaje, se les propuso, haciendo énfasis al eje priorizado de una educación participativa, a madres y padres a compartir en el aula sus experiencias, saberes y conocimientos que le fueron transmitidos de generación en generación. También se coordinó con ellos para realizar las diferentes actividades delegando responsabilidades, para que así todos estuvieran activos desde sus roles.

Es relevante acotar que muchos de los padres de familia sentían vergüenza de hablar con los maestros porque ellos que no entienden bien el quechua por lo que es dificultoso comunicarse. Además, presentaron dificultad para integrarse en actividades como la velada, los desfiles y ferias educativas. A pesar de esta limitación, una de las actividades que abrió las puertas a la comunidad fue durante los juegos plurinacionales donde todos se organizaron, participaron y coadyuvaron en las actividades que requerían y disfrutaron la celebración.

A raíz de este logro, se pudo ayudar a varios estudiantes con problemas de rendimiento académico a través de sus padres. Según el investigador, esta relación ha tenido resultados favorables porque no solamente los docentes son los responsables 
de la educación, sino que este proceso vincula a toda la comunidad, incluyendo a los padres, lo cual se puede apreciar con mayor claridad en el relato a continuación:

En la relación con los Padres de Familia he visto resultados favorables en estudiantes que estaban con bajo rendimiento, fui conversando con los padres, teniendo como instrumentos de apoyo las fichas de seguimiento, y haciéndoles entender que ellos, aunque no sepan leer o escribir pueden influir en la vida de sus hijos/as para que cambien esa realidad, y así algunos lograron aumentar el rendimiento de sus hijos aplazados...

Seguidamente, realizando un análisis de la implementación de MESCP en la unidad educativa, han sido muy importantes los cambios que realizaron los docentes en la práctica pedagógico buscando nuevas estrategias de enseñanza y aprendizaje para sus educandos sumado al compromiso y la voluntad para implementar la nueva ley.

Ya para cerrar este segmento de los resultados, es de gran importancia dar a conocer los diálogos pedagógicos de los cuales surgieron las conclusiones que se presentan más adelante. En estos diálogos se puede apreciar brevemente y con mayor claridad la experiencia que tuvieron los investigadores en este proceso, específicamente con los directivos de las instituciones quienes representaron un punto relevante en los conflictos. Al final se realiza una reflexión sobre el diálogo como estrategia para mermar o disminuir estos problemas.

\section{Diálogos pedagógicos:}

Cinthia Gimena Vargas Cayetano. “...en una reunión administrativa se debería aclarar cuáles son las funciones y las normas que tiene cada miembro de la unidad educativa porque, el director está incumpliendo con las normativas que se deben realizar, como las primeras llamadas de atención y que los docentes estamos dejados en ese aspecto y no puede hacer un descuento respectivo... el director debe ver la realidad del docente, analizar las dificultades..." 
Cesar Vargas Cayetano. "El director es la máxima autoridad de la unidad educativa por eso es importante que sea él mismo quien nos lleve a una buena relación, no solo entre los profesores sino entre todos los que pertenecemos a este ámbito educativo, viendo las situaciones en la que nos presentamos, los problemas que se presentan en la gestión escolar y otros obstáculos que siempre se tienen en todo el año, ver la forma más adecuada y responsable de interceder..."

Orlando Torrico Rocha: "En mi unidad educativa he visto varios problemas en cuanto al gestor. Desde que comenzó su gestión el director es impositor, no acepta las sugerencias de los colegas, no tiene buenas relaciones con los maestros; en algunos casos, cuando se lo saluda no te responde. No hay un espacio de diálogo. El problema inició cuando en sus reuniones nos empezó a tratar de lo peor y en ese momento nadie dijo nada, pero las maestras empezaron a murmurar, lo peor es que el gestor siempre se cree el mejor... Pienso que el diálogo es el medio para recuperar al sujeto en su integralidad junto al maestro y gestor, es muy importante para coordinar diferentes actividades y lograr así integrar a toda la comunidad educativa para la trasformación de la educación, lo que busca la nueva ley Avelino Siñani Elizardo Pérez.

Profesor Cesar Vargas Cayetano: Muy bien continuando con el análisis, el diálogo es un factor importante en nuestra vivencia, ya que el espacio educativo se convierte pues en un espacio para compartir actitudes y acciones básicas de convivencia. Como seres humanos, desde que nacemos convivimos con el medio que nos rodea, en este sentido en la escuela tiene que haber buenas relaciones.

\section{CONCLUSIONES}

$\mathrm{D}$ entro de las problemáticas que se desarrollaron en la experiencia, se concluyó que todas se originaron por factores como: gestores autoritarios con problemas de relacionamiento entre docentes y administrativos, existencia de grupos de profesores con discrepancias, con cierto favoritismo y la falta de trabajo en equipo por parte del plantel docente. Se pudo 
apreciar como problema recurrente el autoritarismo de los actuales gestores dentro el Sistema Educativo Plurinacional.

Tomando en cuenta los aportes que se hicieron en las reflexiones pedagógicas sobre la importancia del diálogo entre los miembros de la comunidad, es de gran relevancia hablar sobre la influencia que este tiene en la solución de los conflictos. Gracias al diálogo se permite la movilización a la acción y al compromiso como práctica de liberación y transformación. Este aspecto va a propiciar la convivencia entre las culturas, los maestros y estudiantes mediante relaciones ética basada en el respeto a las diferencias. Es por tanto que, el diálogo como estrategia para fortalecer y establecer espacios de relacionamiento, participación y transformación en la gestión educativa, es uno de los elementos clave para la convivencia.

Otro aspecto relevante que se puede concluir de esta investigación es sobre el perfil del gestor o director de la unidad educativa. Debería seleccionarse a un personal capacitado con conocimientos en relaciones humanas ya que uno de los principales problemas que se observó en ambas instituciones fue en torno a la figura del director. Esto se evidencia en los siguientes relatos, el primero de Orlando Torrico Rocha y el segundo de Cesar Vargas Cayetano quienes coinciden en que el Gestor educativo es uno de los principales responsables de propiciar un ambiente armónico en la institución.

Orlando Torrico Rocha.

Considero importante que el gestor focalice las necesidades detectadas en la comunidad, a través de sus docentes, construir con ellos un espacio de trabajo armónico, donde sea posible una convivencia escolar positiva... necesitamos gestores más humanos, que siempre busquen rescatar al sujeto, y que los sujetos no estén por encima de la ley o simples informes, sino que los sujetos sean la priorización siempre...

Cesar Vargas Cayetano:

El director es la máxima autoridad de la unidad educativa, por eso es importante que sea él mismo que nos lleve a una buena relación no solo entre los profesores sino entre todos los que pertenecemos a esta ámbito educativo, viendo las situaciones en la que nos 
presentamos, los problemas que se presentan en la gestión escolar y otros obstáculos que siempre se tiene en todo el año, ver la forma más adecuada, responsable y justa para cualquier sanción que se hubiera y también cualquier malentendido con cualquier docente, sea antiguo o nuevo, sea hombre o mujer...

Pero un gestor educativo formado en el MESCP, no solo debe garantizar mejores relaciones institucionales o superar ciertas formas de trabajo como el control de profesores, niños/as, administrativos, PPFF, etc., sino que también debe articular conocimientos entre los mismos actores educativos con el fin de potenciar y lograr una transformación a la diversidad de problemas que atañen a la institución. En palabras de los investigadores:

Nos llama la atención, sobre todo, la focalización en la construcción colaborativa, y como está cambiando la visión autorreferencial que se tenía de la escuela, el gestor no puede producir el cambio solo, sino que su rol fundamental es crear las condiciones para que dicho cambio se produzca

Se considera entonces que la función del gestor y de los docentes, debe orientarse a mantener un comportamiento mucho más asertivo, brindando apoyo y colaboración a los padres, estudiantes y autoridades, fundamentado en el reglamento, pero también realizando un análisis de la realidad en cada problemática ya que, el contexto influye en la vida de cada quien de formas distintas.

De este modo, para transformar la gestión educativa es necesario interpretar el contexto asumiendo las experiencias en el ámbito laboral, y así asumir de manera crítica y reflexiva las dificultades que se puedan presentar; fortalecer la participación de la comunidad educativa comenzando por los maestros, la construcción de una realidad que permita transformar las relaciones sugeridas por el modelo educativo socio comunitario productivo, y así lograr una educación participativa.

Por esta razón los docentes deben estar comprometidos como parte de la comunidad educativa, a generar acciones orientadas al logro de los objetivos planteados en la institución donde trabajan. Además, favorecer el trabajo en equipo, las buenas relaciones, la 
potenciación del sujeto a sujeto, lo cual genera un mayor compromiso con los padres de familia, con los estudiantes y comunidad educativa en general apuntando a un clima institucional de diálogo colaborativo y reflexivo.

A modo de cierre, se presentan algunos diálogos reflexivos de las percepciones que tuvieron los investigadores al culminar este trabajo:

Cinthia Gimena Vargas Cayetano. Para terminar, pienso que nosotros los docentes, aparte de ser individualistas no nos gusta ver que otro haga un trabajo mejor, nosotros como docentes no pensamos en mejorar, nos conformamos con lo que tenemos y hacemos y si vemos que otro es protagonista tal vez de una actividad, nos genera molestia. Respecto al director, lo que se ha conversado es que su actitud decepciona bastante, estos profesores nunca van a cambiar, son profesores que se han enraizado en el individualismo, lo único que se puede hacer es esperar que salgan de la comunidad de alguna manera, si bien él dice que está trabajando... el verá.

Cesar Vargas Cayetano. Tras todas estas experiencias vividas, claro con la aplicación modelo educativo socio comunitario productivo, puedo concluir que este modelo educativo responde a los enfoques de los campos educativos al transformar la práctica misma y su visión metodológica en situaciones concretas de la realidad.

Orlando Torrico Rocha. La experiencia vivida en el proceso de formación del diplomado fue de mucha relevancia porque me permitió, valorar, reflexionar de manera crítica y cambiar la forma de mirar mi práctica educativa y tener una postura propositiva sobre los procesos de hacer una buena gestión enmarcadas en las buenas relaciones que favorezcan la construcción de la institución y el logro de posibilidades planteadas en la ley 070, Avelino Siñani - Elizardo Pérez.

\section{REFERENCIAS}

Gaceta Oficial de Bolivia (2007). Plan Nacional de Desarrollo "Bolivia digna y soberana, productiva y democrática para vivir bien" La Paz-Bolivia

Gaceta Oficial del Estado Plurinacional de Bolivia (2010). Ley No. 070 Ley de la educación "Avelino Siñani - Elizardo Pérez" 
Ministerio de Educación (2012). "Currículo Base del Sistema Educativo Plurinacional", documento de trabajo, La Paz-Bolivia Ministerio de Educación (2012). Programa de Formación Complementaria para Maestras y Maestros en Ejercicio (PROFOCOM) La Paz-Bolivia

Ministerio de Educación (2017). Diplomado en Formación para la Transformación de la Gestión Educativa en el Modelo Educativo Sociocomunitario Productivo (2da. Versión) La PazBolivia

Ministerio de Educación, Vice ministerio de Educación Regular. (2012) "Currículo del Subsistema de Educación Regular", documento de trabajo, La Paz-Bolivia 